


\title{
Surface Effects on Lyotropic Liquid Crystals
}

\author{
Elisabeth Andreoli de Oliveira \\ Instituto de Física de Universidade de São Paulo, \\ Caixa Postal 66318, CEP 05389-970, São Paulo, São Paulo, Brasil
}

Received 08 September, 1998

\begin{abstract}
Liquid crystals are very sensitive to surface effects. In fact $\Gamma$ these effects are very useful in designing eletro-optical devices. We present a review of the theoretical models that describe the surface interactions in liquid crystals $\Gamma$ focusing on lyotropic systems. Experimental results will be presented and compared to theoretical predictions.

PACS number(s):61.30.-vГ61.30.GdГ64.70.MdГ
\end{abstract}

\section{Introduction}

Liquid crystals mesophases are present in materials composed of interacting molecules or aggregates of molecules with shape anisotropy. These materials can be divided in two categories according to the relevant parameter in the phase transitions; the thermotropic and lyotropic liquid crystals. In thermotropics $\Gamma$ the building blocks are molecules $\Gamma$ and the phase transitions are driven by variations of temperature. Lyotropic systems are obtained by the dispersion of amphiphilic molecules in water $\Gamma$ that assembly in anisotropic aggregates called micelles. The micelles can be seen as biaxial objects $\Gamma$ with typical dimensions of $(100 \times 70 \times 30) \AA$. The phase transitions in lyotropics occur by temperature or concentration variations.

The mesophases are classified according to the symmetry they exhibit. The simplest mesophase is the nematic phase $\Gamma$ consisting of elongated molecules (or aggregates) that tend to align parallel to each other $\Gamma$ with a long range orientational order but no long range positional order. The average molecular orientation defines a vector $\mathbf{n} \Gamma$ called the director. More complex mesophases can present some degree of positional order such as in the smectic phase where the molecules are organized in layers with one dimensional long range positional order[1].

The orientation of the director can be fixed by an external field or by the surfaces effects. The existence of a limiting surface introduces a perturbation in the order of the molecules imposing some preferential orientation that propagates to the bulk by means of elastic interactions. There are experimental evidences of formation of smectic layers in the nematic phase close to the nematic-solid and nematic-air interface $\Gamma$ exhibiting both orientational and positional order[2Г3]. This can be related to the break of the translational invariance of the molecules in the nematic phase in the limiting surface. Molecular dynamics simulation show that a rough surface induces a smetic layering within a few molecular lengths in a uniaxial nematic liquid crystal. Moving towards the bulk the positional order desapears $\Gamma$ remaining only the orientational order along the direction fixed by the surface [4].

Magnetic or electric fields can be used to induce the orientation of the director; the director tends to align parallel to the direction of the field $\Gamma$ if the anisotropy of the diamagnetic (or electric) susceptibility is positive. However $\Gamma$ close to the boundaries there is a competition between the orientation imposed by the surface and the orientation imposed by the field $\Gamma$ which results in a distorted state. When the field is removed $\Gamma$ the orientation imposed by the boundary surfaces propagates to the bulk and the orientation of the director is fixed by the surface. These effects are widely used to produce eletro-optical devices $\Gamma$ like flat panels and displays[5]. Usually $\Gamma$ for thermotropic liquid crystals the interactions of the molecules with the substrate are stronger than the others interactions $\Gamma$ what is known as strong anchoring. In the surface layer there are only 
small distortions in the orientation of the molecules $\Gamma$ and when the field is removed the orientation imposed by the substrate is dominant and propagates to the sample. Under the action of a magnetic field $\mathrm{H} \Gamma$ the switching time is $\tau_{v}=\eta /\left(\chi_{a} H^{2}\right)$, where $\eta$ is the rotational viscosity and $\chi_{a}$ is the anisotropy of the diamagnetic susceptibility[1]. For applied electric fields $(\mathrm{E}) \Gamma \chi_{a}$ is replaced by the anisotropy of electric susceptibility $\Gamma$ and $\mathrm{H}$ is replaced by $\mathrm{E}$.

\section{Surface effects}

The interactions of the liquid crystal with the boundary surfaces are very complex and are far from being completly understood[6]. It has been proposed that the surface interactions can be described $\Gamma$ in a phenomenological approach $\Gamma$ by an anisotropic energy $F_{S}$ that is usually characterized by an anchoring strengh $w$ and an easy-axis $\mathbf{n}_{0} \Gamma$ which corresponds to the preferential orientation of the nematic phase Timposed by the boundary surface $\Gamma$ in the absence of bulk distortions. The simplest expression of $F_{S}$ was proposed long ago by Rapini and Papoular [7]. According to these authors $F_{S}$ is given by $F_{S}=-(w / 2)\left(\mathbf{n} . \mathbf{n}_{0}\right)^{2} \Gamma$ with typical values of $w$ in the range of $10^{-3}$ to $10^{-1} \mathrm{erg} / \mathrm{cm}^{2}$ Tfor thermotropic liquid crystals.

The validity of this Rapini-Papoular expression has been contested by some authors; it is argued that the correct expression of $F_{S}$ should contain terms of higher order in the expansion $\Gamma$ only of even-order due to symmetry $\mathbf{n}$ and $-\mathbf{n}[8 \Gamma 9 \Gamma 10 \Gamma 11]$. However $\Gamma$ other authors suggest that the contribution of polar interactions at the interface should also be taken into account $[12 \Gamma 13 \Gamma 14] \Gamma$ which would include odd terms in the expansion. There is also some discussion about the interpretation of experimental results [15]; it was shown that if the flexo-electric effect arising from distortion in the bulk is taken into account $\Gamma$ a good accordance with the Rapini-Papoular expression is obtained. Other effects can be present $\Gamma$ like adsoprtion of ions at the boundary surfaces $\Gamma$ resulting in an electric field $\Gamma$ that interacts with the nematic in the surroundings. The difficulties concerning the interpretation of experimental results are due to the fact that many effects must be taken into account $\Gamma$ some of them related to bulk effects that cannot be experimentally separated.
It has been recently shown that lyotropic liquid crystals exhibit anchoring properties very different with respect to thermotropic liquid crystals. Two orientation processes have been observed under the action of a magnetic field; a fast one related to the orientation of the director in the bulk $\Gamma$ parallel to HГand a slow one $\Gamma$ with a characteristic time $(\tau)$ about $10^{2}$ larger than the bulk one. This slow process has been related to the orientation of the director in the surface layer [16Г17] and it was found that $\tau$ is proportional to $\mathrm{H}^{-2}$. If the surface orientation process is completed $\Gamma$ the final orientation is uniform $\Gamma$ with the director parallel to the field. In this situation $\mathrm{H}$ can be removed $\Gamma$ without any significant change of orientation of the sample $\Gamma$ and the final state is stable.

A qualitative description of the structure of the surface layer can be obtained in analogy to thermotropic liquid crystals. In the surface layer $\Gamma$ due to break of translational symmetry $\Gamma$ the amphiphilic molecules would assembly in a bilayer structure $\Gamma$ like in a lamellar phase. There are experimental evidences that the bilayer $\Gamma i n$ the lamellar phase is not continous $\Gamma$ but consists rather of amphiphilic islands surrounded by water [18]. Within this picture one can imagine that reorientation of the surface layer $\Gamma$ consists of the reorientation of the islands parallel to the magnetic field. Since they are bigger than the micelles $\Gamma$ the effective viscosity should be larger than in the bulk $\Gamma$ what would explain the large response time. This reorientation process has been known as the gliding of the surface layer.

In a recent experiment $\Gamma$ a Freederickz transition was used to estimate the anchoring energy of a lyotropic system in a planar configuration $\Gamma$ assuming that the surface energy is described by the Rapini-Papoular expression. The anchoring strenght $w$ Tobtained from the experiment is about $10^{-3} \mathrm{erg} / \mathrm{cm}^{2}[19] \Gamma$ which is consistent with a weak anchoring. This description is not completly satisfactory $\Gamma$ because according to this $\Gamma$ there should be a relaxation process by removing the magnetic field $\Gamma$ due to the surface energy. However $\Gamma i t$ gives some idea of the magnitude of the surface interaction.

In this paper we will review the theoretical models proposed to explain both the dynamical behavior and the equilibrium states. The predictions of the models will be compared to experimental results obtained for lyotropic samples in the uniaxial and biaxial nematic 
phases.

\section{Experimental techniques}

The lyotropic sample consists of a mixture of potassium larurate $(\mathrm{LK}) \Gamma$ decanol $(\mathrm{DeOH})$ and water $\Gamma$ that presents a rich phase diagram $\Gamma$ with uniaxial and biaxial nematic phases. In this paper we refer to experimental results obtained with different compositions and sequence of phases. The characteristic of the phases (uniaxial calamitic or discotic or biaxial) is determined by measuring the birefringence as a function of the temperature[20].

The samples are encapsulated in flat microslides (Vitrocom) $\Gamma 200 \mu m$ thick and $4 \mathrm{~mm}$ wide. The inner surfaces of these microslides were examined in a interferential microscope and it was observed that surfaces are plane $\Gamma \operatorname{smooth} \Gamma$ and without irregularities greater than $012 \mu \mathrm{m}$. No surface treatment was applied.

The reorientation process is observed by measuring the transmittance of the sample between crossed polarizers $\Gamma$ as a function of the time $\Gamma$ when the magnetic field is applied. The orientation of the polarizers is chosen to have the maximum of transmittance at $t=0$, when the director is parallel to the $x$ axis. The direction of the magnetic field makes an angle $\theta=45^{\circ} C$ with the $x$ axis. The experimental setup is described in detail in ref[17].

The experimental curves of transmittance are compared to calculated curves for some profile of the director. The sample is assumed as a non-homogeneous optically anisotropic medium $\Gamma$ that can be divided into many thin layers $\Gamma$ with a uniform orientation in each layer. The propagation of the ligth through this medium can be calculated using Jones matrix $\Gamma$ where in each layer there is a phase shift between the ordinary and extraordinary rays $\Gamma$ and a rotation of the direction of the polarization of the light[17].

\section{Dynamic behavior of the sur- face layer}

Lyotropic liquid crystals are composed of biaxal micelles $\Gamma$ that can undergo simultaneously shape and orientational variations due to variations of temperature or concentration. In the biaxial phase one can define three directors $\Gamma$ which coincide wiht the symmetry axes of the micelles. The two uniaxial nematic phases can be explained by orientational fluctuations. One of the particular features of lyotropic liquid crystals is the possibility of the gliding of the director in the surface layer. Let us first analyse the factors that can influence the surface reorientation process in both thermotropic and lyotropic liquid crystals.

The order parameter in thermotropic liquid crystal increases continuously from the bulk to the surface $\Gamma$ which is usually called excess surface order. In addition $\Gamma$ the surface viscosity is higher than the viscosity in the bulk. In this case $\Gamma$ the surface is strictly twodimensional $\Gamma$ and although the magnetic and the elastic torques have the same order of magnitude Conly the elastic torque acts on the surface[1]. Then the action fo the magnetic field on the surface is indirect $\Gamma$ (by means of the twist distortion in the bulk) $\Gamma$ thus $\tau$ would be proportional to $1 / H$. But in a lyotropic liquid crystal this result is in contradiction with the experimental results[17]. Strong anchoring of the micelles at the surface leads to the formation of a surface layer with an order different with respect to the order in the bulk. Due to the anchoring $\Gamma$ the symmetry plane of the surface fixes the orientation of one of the directors $\Gamma m a k-$ ing the order of the surface layer biaxial. One director is always perpendicular to the susbstrate and the two others can rotate in the plane. Therefore $\Gamma$ there is a surface layer $\Gamma$ of finite thickness and two-dimensional orientational order. Because of its finite thickness $\Gamma$ the surface layer can interact directly with the magnetic field $\Gamma$ in addition to the elastic interaction $\Gamma$ that takes place mainly in the boundary which separates the biaxial interface from the bulk. It creates an elastic torque between the two parts of the liquid crystal with different orderГso it is much smaller than the elastic torque in thermotropic liquid crystals. This leads to a twist of the director in the bulk $\Gamma$ which is much less pronounced than it would be for a thermotropic.

In the bulk the order parameter is the threedimensional second rank tensor $Q_{i j}$. Let us call $r \Gamma$ the modulus of the nematic order parameter in the bulk $\Gamma$ which depends only on the temperature. Thus the components of $Q_{i j}$ are $\eta_{2}=r e^{i \ell \varphi} \Gamma$ and $\eta_{-2}=r e^{-i \ell \varphi}$ where $-2 \leq \ell \leq 2$ and $\varphi$ is the angle between the director in the bulk and the $x$ axis[21]. The order in 
the surface layer is described by the tensor $\mathrm{q}_{i j} \Gamma$ with spherical components $\eta_{s}=q_{x x}-i q_{x y}=\rho e^{i 2 \Phi} \Gamma$ and $\eta_{s}^{*}=q_{x x}+i q_{x y}=\rho e^{-i 2 \Phi} \Gamma$ where $\rho$ is the modulus of the nematic order parameter in the surface layer $\Gamma$ and $\Phi$ is the angle between the orientation of the director in the surface layer and the $x$ axis. This tensor has two orthogonal eigenvectors; $\mathbf{e}^{+}$and $\mathbf{e}^{-} \Gamma$ which represent the two directors of the biaxial surface layer $\Gamma$ in the plane parallel to the boundary surface.

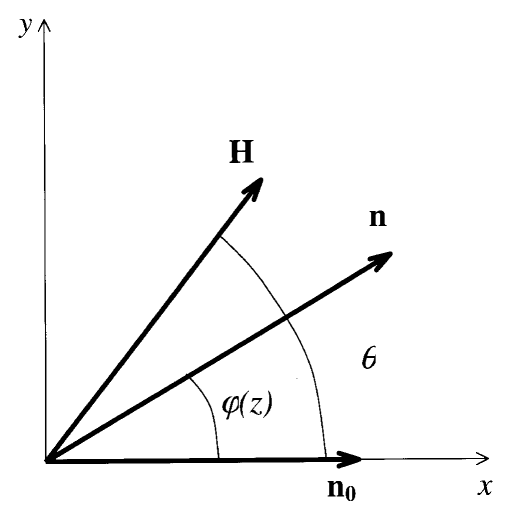

Figure 1: A planar nematic liquid crystal sample, homogeneously oriented along the $x$ axis at $t=0$. The magnetic field is applied at angle $\theta$ with respect to the $\mathrm{x}$-axis. In the twisted state $\varphi(z)$ is the angle between the director $\mathbf{n}$ and the $x$-axis. At the surface layer the orientation of the director is given by the angle $\Phi$ which does not depend on $\mathrm{z}$, but only on the time $t$.
Let us consider a semi infinite sample $\Gamma$ with a boundary surface located at $z=0$. At $t=0$ the director is oriented along the $x$ axis and a magnetic field $\mathbf{H}$ is applied parallel to the boundary surface $\Gamma$ making an angle $\theta$ with respect to $x$ axis (Fig. 1). The initial orientation corresponds to $\mathbf{n}$ parallel to the eigenvector $\mathbf{e}^{+} \Gamma$ which corresponds to the direction of the biggest dimension of the amphiphilic aggregates. Since the reorientation of the director in the bulk is much faster than in the surface layer $\Gamma$ we will consider that at $t=0$ the director in the bulk is parallel to $\mathbf{H}$. With the time the surface layer will glide to a new equilibrium orientation $\Gamma$ given by $\Phi(t)$. It is important to note that $\Phi$ depends only on the time $\Gamma$ and does not depend on $z$. Near the boundary with the surface layer the twist appears with the limit angle $\varphi(z \rightarrow 0)=\varphi_{0} \neq 0$.

The free energy of the system $\Gamma$ consists of the free energy of the surface layer $\left(F_{S}\right)$ and the free energy of the bulk due to the distortion introduced by the magnetic field $\left(F_{V}\right)$. We introduce also a contribution due to the coupling between the order of the bulk and surface layer $\Gamma\left(F_{S V}\right)$. Then we can write the free energy per unit area as:

$$
\begin{aligned}
F= & F_{V 0}+\int_{0}^{\infty}\left[\frac{K}{2}\left(\frac{d \varphi}{d z}\right)^{2}+\frac{1}{2} \chi_{a} H^{2} \int_{0}^{\infty} \cos ^{2}(\theta-\varphi)\right] d z+ \\
& F_{S 0}-\frac{1}{2} D H^{2} \rho \cos ^{2} \Phi-\operatorname{Cr} \rho \cos ^{2}\left(\Phi-\varphi_{0}\right)
\end{aligned}
$$

where $F_{S 0}$ and $F_{V 0}$ are the free energies of the non perturbated state and $K$ is the elastic constant of the liquid crystal. The last term in eq. 1 represents the coupling of bulk and the surface layer Tinvolving both $r$ and $\rho$. The coupling constant $C$ can be either positive or negative. The surface free energy is due to the direct interaction of the surface layer with the magnetic field $\Gamma$ by means of a coupling constant $D \Gamma$ positive.

The orientational dynamics of the system is described by Landau-Khalatnikov equations $\Gamma$ in other terms by the balance of magnetic $\Gamma$ elastic and viscous torques acting on the bulk and on the interface;

$$
\begin{gathered}
K \frac{d^{2} \varphi}{d z^{2}}=\chi_{a} H^{2} \sin (\theta-\varphi) \cos (\theta-\varphi), \\
C r \rho \sin 2\left(\varphi_{0}-\Phi\right)=-K\left[\frac{d \varphi}{d z}\right]_{z \rightarrow 0}, \\
-\gamma \frac{d \Phi}{d t}=\frac{1}{2} D \rho H^{2} \sin 2 \Phi+C r \rho \sin 2\left(\Phi-\varphi_{0}\right),
\end{gathered}
$$

where $\gamma$ is the rotational viscosity of the surface layer. Eq. 2 corresponds to the balance of the torques in the 
bulk and eq. 3 to the balance of the torque in the boundary of the bulk and surface layer. The last equation represents the reorientation of the director in the surface layer $\Gamma$ where the elastic torque and magnetic torque are equilibrated by the viscous torque. From eq. 2 Tone obtains;

$$
\frac{d \varphi}{d z}=\frac{1}{\xi}\left[\cos ^{2}\left(\theta-\varphi_{\infty}\right)-\cos ^{2}(\theta-\varphi)\right]^{1 / 2},
$$

where $\xi^{2}=\left(K / \chi_{a}\right) / H^{2} \Gamma$ is the magnetic coherence length and $\varphi_{\infty}=\varphi(z \rightarrow \infty)$. Since far from the boundary surface $\Gamma$ the director is parallel to the magnetic field $\Gamma$ $\varphi_{\infty}=\theta$. The profile of the director in the bulk $\Gamma$ is obtained by integration eq. $5 \Gamma$ with the proper boundary conditions in the interface; $\varphi(z \rightarrow 0)=\Phi$. This gives;

$$
\frac{\varphi}{2}=\frac{\theta}{2}-\arctan \left[\exp \left(-\frac{z}{\xi}\right) \tan \left(\frac{\theta-\Phi}{2}\right)\right]
$$

In eq. 5Ttaking the limit for $z \rightarrow 0$, and substituting in eq. 3 Гit results that;

$$
C r \rho \sin 2\left(\varphi_{0}-\Phi\right)=-\frac{K}{\xi} \sin \left(\theta-\varphi_{0}\right) .
$$

Assuming small deformations in the surface layer and in the boundary to the bulk $\Gamma$ the above equation can be linearized and solved to find $\varphi_{0}=\varphi_{0}(H)$;

$$
\varphi_{0}=\frac{\Phi-\lambda \theta}{1-\lambda}
$$

where $\lambda=H / H_{0}$ and $\mathrm{H}_{0}=\sqrt{K \chi_{a}} /(2 C r \rho)$.

The dynamical behavior of the surface layer can then be obtained linearizing eq. 4 and with the calculated value of $\varphi_{0}$. This leads to the following diferential equation:

$$
-\gamma \frac{d \Phi}{d t}=\left[D \rho H^{2}-2 C r \rho \frac{\lambda}{1-\lambda}\right] \Phi+2 C r \rho \frac{\lambda \theta}{1-\lambda},
$$

which can be easily integrated to obtain the orientation of the director in the surface. Imposing the proper boundary conditions Tone can write the solution;

$$
\Phi(t)=\theta\left(1-e^{-t / \tau}\right),
$$

where $\tau$ is the characteristic time of the reorientation of the surface layer. The expression for $\tau$ can be simplified to show the dependence on $\mathrm{H}$ :

$$
\frac{1}{\tau}=\frac{1}{\tau_{0}}\left[\frac{H^{2}}{H_{t}^{2}}-\frac{H}{H-H_{0}}\right]
$$

with $1 / \tau_{0}=2 C r \rho / \gamma$ and $H_{t}^{2}=2 C r / D$. For large fields $\Gamma$ $1 / \tau$ is proportional to $\mathrm{H}^{2}$ and the contribution of the second term in eq. 11 can be neglected. The meaning of the $\mathrm{H}_{0}$ and $\mathrm{H}_{t}$ are illustrated in Fig. 2. For $\mathrm{C}>0$ and $\mathrm{D}>0 \Gamma 1 / \tau_{0}$ is positive and $H_{t}$ is real.

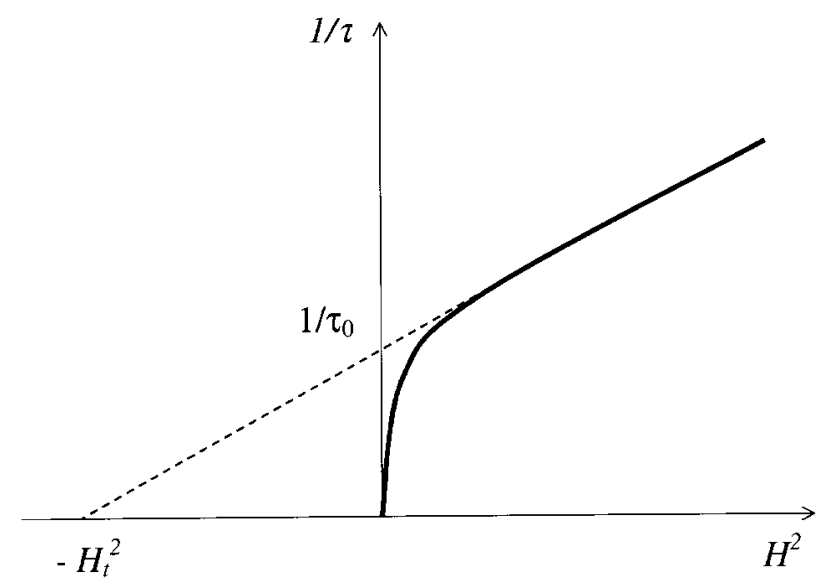

Figure 2: Dynamics of the gliding director in the biaxial surface layer. The model predicts that $1 / \tau$ is proportional to $\mathrm{H}^{2}$, for high fields. The values of $H_{0}^{2}$ and $H_{t}^{2}$ are related to the phenomelogical constants of the free energy expansion, for $C>0$ and $D>0$.

An interesting behavior is obtained if we consider the case when the elastic interaction with the bulk and the magnetic torque try to orient the interface director in different directions. This happens when $\mathrm{C}<0$ and $\mathrm{D}>0$. The magnetic torque tries to orient $\mathbf{e}^{+}$parallel to $\mathrm{H}$ एwhile the elastic interaction with the bulk tries to orient $\mathbf{e}^{-}$parallel to the magnetic field. This situation may occur in the bulk biaxial phase $\Gamma$ where all the three bulk directors $\mathbf{n}_{\mathbf{i}}$ are ordered.

The characteristic time of the surface reorientation process was experimentally determined by transmittance measurements in uniaxial calamitic and biaxial nematic phases. In the analysis of the experimental curves of transmittance it was assumed that the sample was uniformly oriented parallel to the magnetic field $\Gamma$ except in the thin boundary layers of thickness $\xi$. In this simple three layers model $\Gamma$ the orientation of the director in the surface layers was considered to follow the eq. 10[17]. The experimental values are shown in Fig. $3 \Gamma$ in the nematic calamitic (a) and biaxial phases $(b) \Gamma$ where $T_{c}$ is the temperature of the calamitic to biaxial phase transition in the bulk. The calculated values of $1 / \tau_{0}$ and $\mathrm{H}_{t}^{2}$ for different temperatures in the uniaxial and biaxial phases cleary indicates the change of 
the dynamical behavior $\Gamma$ as is shown in Fig. 4. When the temperature is decreased towards the biaxial phase $\Gamma$ both parameters change sign.

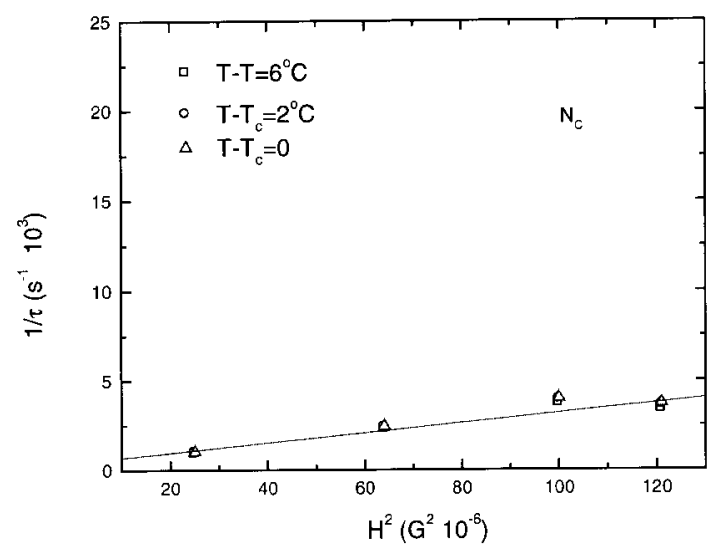

(a)

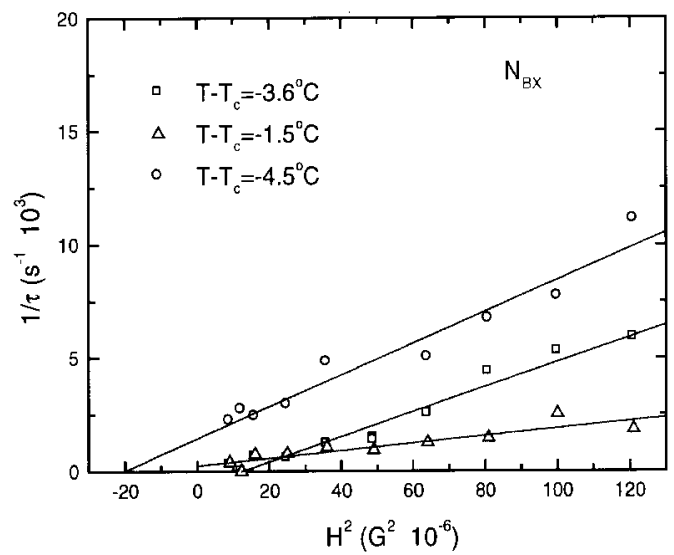

Figure 3: Experimental values of the characteristic time for the surface reorientation process. a) Lyotropic sample in the calamitic and $b$ ) in the biaxial nematic phases. $T_{c}$ is the temperature of the calamitic to biaxial nematic phase.

A detailed investigation of the reorientation of the surface layer in the calamitic phase in the region of very low fields revelead a striking behavior. The simple three layers model for the profile of the director is suitable for high fields $\Gamma$ but below a certain value it cannot be used to fit the experimental curves of transmittance. Then $\Gamma$ the fitting was perfomed taking the profile described by eq. 6. and allowing the gliding of the surface layer; $\Phi=\Phi(t)$. In the fitting it was considered the possibility that the final orientation of the surface could be different from the one imposed by the magnetic field.
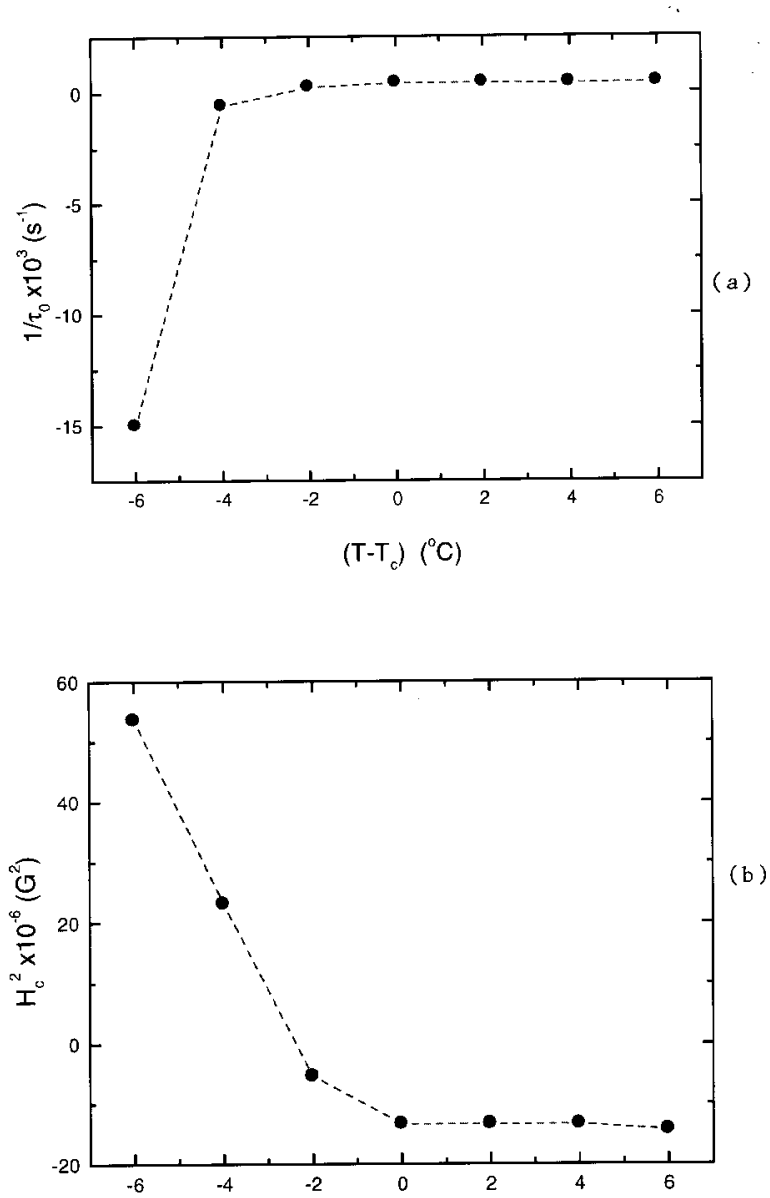

$\left(\mathrm{T}-\mathrm{T}_{\mathrm{c}}\right)\left({ }^{\circ} \mathrm{C}\right)$

Figure 4: Dependence of the parameters (a) $1 / \tau_{0}$ and (b) $\mathrm{H}_{t}^{2}$ on the temperature.

$$
\Phi(t)=\Phi_{f}\left(1-e^{-t / \tau}\right)
$$

where $0<\Phi_{f}<\theta$ and $\Phi_{f}=\Phi(t>>\tau)$ gives the final orientation of the director in the surface.

The experimental values of $\tau$ as a function of $\mathrm{H}$ are presented in Fig. 5. In a log-log plot $\Gamma$ we notice that are two lines $\Gamma$ with different inclinations $\Gamma$ changing the behavior for $H \approx 5000 G$. For fields below this value $\Gamma$ the curves of transmittance were fitted with a continous profile of the director $\Gamma$ given by eq. 6 (arctan profile) resulting in $\tau$ proportional to $H^{-2}$. For fields higher than $5 k G \Gamma$ the fitting was possible only with the discontinous profile of the director (three layers model). In this case $\Gamma$ it is observed that $\tau$ is proportional to $H^{-3 / 2}$. We supose that for high fields $\Gamma$ there is a breaking of the elastic coupling between the surface layer and the bulk $\Gamma$ and the theory developed above would no more be valid. 


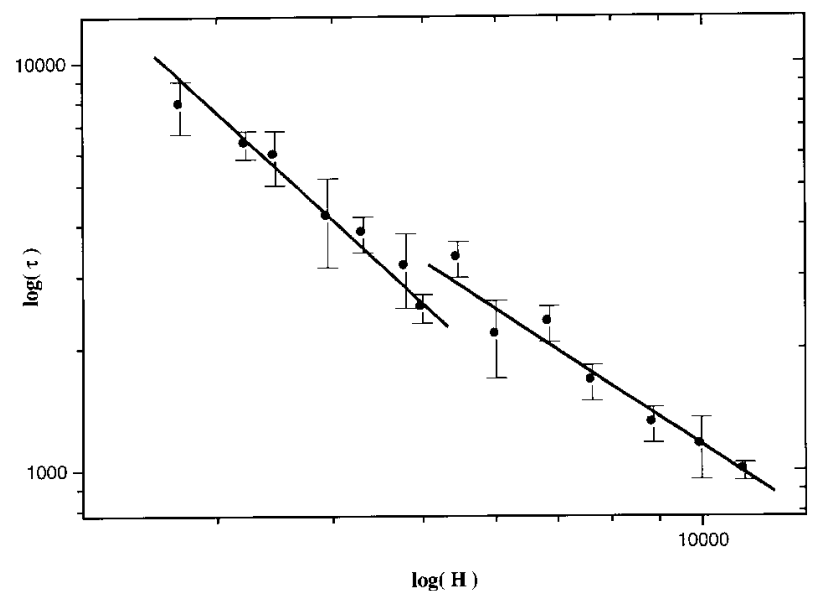

Figure 5: Experimental values of $\tau$ (surface reorientation time) as a function of $\mathrm{H}$, for a nematic sample in the calamitic nematic phase. It is noticeable the change in the dynamical behavior for $H \approx 5000 G$. The inclination is different in each branch of the line.

\section{Equilibrium states}

We will consider that in the equilibrium states there is no discontinuity in the orientation in the boundary that separates the surface layer and the bulk $\Gamma$ this means that the twist is continous and $\varphi_{0} \rightarrow \Phi$. However $\Gamma$ if the final state is distorted $\Gamma$ it means that the elastic torque is being equilibrate by a surface torque.

Let us consider the situation where the director in the surface layer is oriented along the direction $\mathbf{n}_{0}$ and there is a torque $\Gamma_{e} \Gamma$ that tends to align the director parallel to the magnetic field. $\Gamma_{e}$ is the elastic torque; $\Gamma_{e}=K(d \varphi / d z)$ एwith the value of $d \varphi / d z$ given by eq. 5 . The reorientation of the director at the surface strongly depends on the kind of coupling originating the surface orientation. If the anchoring is strong $\Gamma$ the average orientation of the director at the surface will remain parallel to $\mathbf{n}_{0}$ for any applied torque. HoweverГif the anchoring is weak $\Gamma$ and $H$ is larger than a critical value the torque applied by the field is able to reorient the director to break the physical "bonds" between the molecules of the liquid crystal and the substrate.

This phenomenon is similar to the dry-friction of a body on a solid substrate $\Gamma$ if a force is applied to the body it starts to move when the applied force becomes larger that a critical value[22]. This critical value depends on the nature of the two bodies in contact $\Gamma$ and due to its statistical nature $\mathrm{Cit}$ does not depend on the position of the body. If the body is moving and the applied force is removed $\Gamma$ it will stop $\Gamma$ without returning to its original position. According to this picture the reorientation of the director in the surface layer of the lyotropic liquid crystal is similar to the dry friction phenomenon. In this case ${ }^{2}$ instead of a critical force $\Gamma$ we are dealing with a critical torque that must be applied to break the molecular interactions between the substrate and the liquid crystal medium. If the elastic torque is smaller than the critical torque the orientation of the director at the surface is $\Phi=0$ Гif it is larger $\Gamma$ then the orientation of the director in the equilibrium will be obtained from the balance of the elastic and "dry friction $"$ torque $\left(\Gamma_{d}\right)$ Гexpressed as;

$$
\frac{K}{\xi}\left[\cos ^{2}\left(\theta-\varphi_{0}\right)-\cos ^{2}(\theta-\Phi)\right]=\Gamma_{d} .
$$

The critical condition is obtained when $\Gamma_{e}(H)=\Gamma_{d} \Gamma$ and this occurs for a certain field $\Gamma H_{C}^{*} \Gamma$ that is called the surface critical field. For $H=H_{C}^{*} \Gamma \Phi=0$, and we will assume that in the bulk the director is parallel to $\mathbf{H}$; $\varphi_{\infty}=\theta$. With these assumptions in eq.13 Гone obtains the threshold condition;

$$
\frac{K}{\xi^{*}} \sin \theta=\Gamma_{d}=\Gamma_{c}
$$

which defines the value of $H_{C}^{*}$;

$$
H_{C}^{*}(\theta)=\frac{\Gamma_{d} \sin \theta}{\sqrt{K_{\chi_{a}}}} .
$$

If the value of $H_{C}^{*}$ is experimentally determined $\Gamma$ one calculates the "dry torque "from the above equation. Taking $\Gamma_{d}$ given in eq. $14 \Gamma$ the orientation of director in the surface is then calculated $\Gamma$ as a function of $H$;

$$
\Phi=\theta-\arcsin \left[\frac{H_{C}^{*}(\theta) \sin \theta}{H}\right]
$$

The measurement of the transmittance $\Gamma$ as described in the previous sections $\Gamma$ allows the determination of $\tau$ and $\Phi$ as a function of $H$. The experimental results are shown in Fig. 5 (for $\tau$ ) and in Fig. 6(a) $\Gamma$ for $\Phi$ as a function of $H$. Each point corresponds to an average of several measurements $\Gamma$ with an uncertainty of $2^{\circ} \mathrm{C}$ in the value of $\Phi$. The value of $\Phi$ starts to be different from zero for $H \approx 1000 G$, increasing fast and tending to a saturation regime $\Gamma$ but with a jump for $H \approx 5000 G$. For the same value of $H$ there is a discontinuity in the behavior of $\tau$ (Fig. 5). 

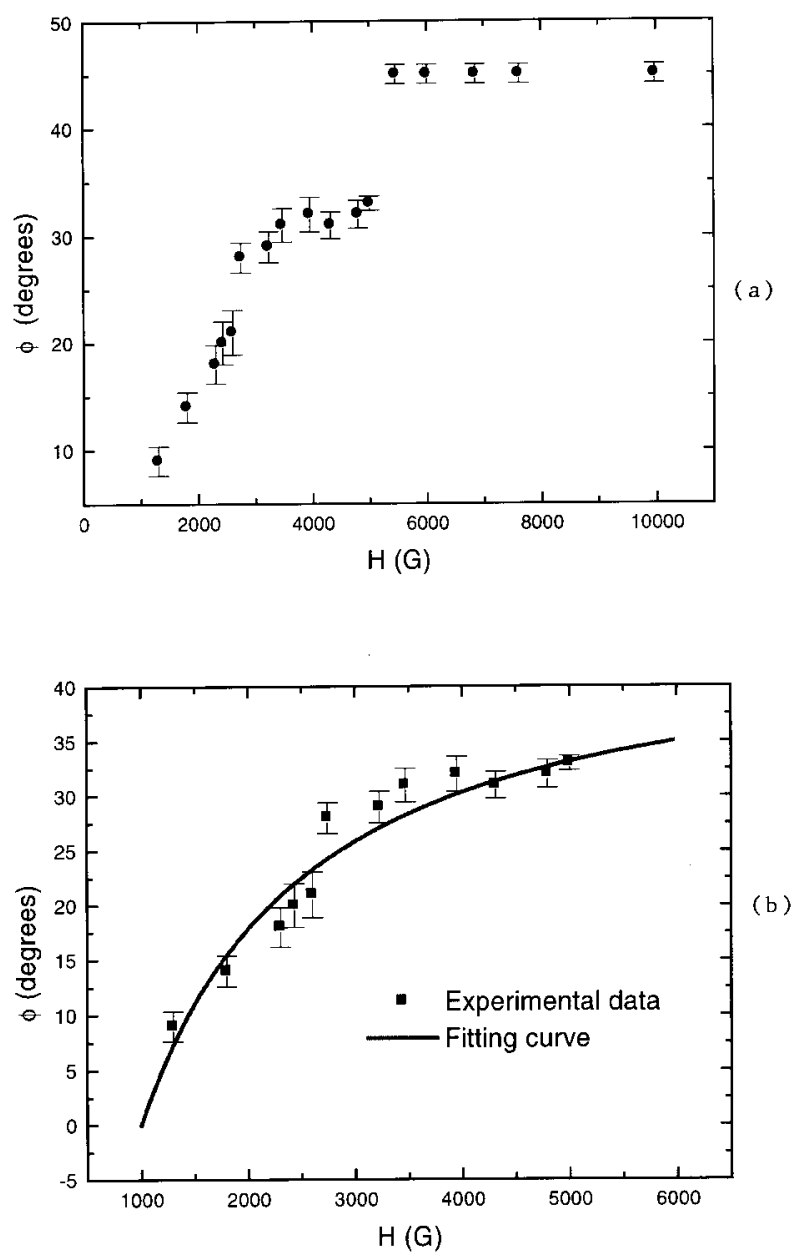

Figure 6. (a) Final orientation of the director in the surface layer for a lyotropic nematic sample in the calamitic nematic phase. (b) The solid curve corresponds to the fitting obtained from eq. 19, assuming $H_{C}^{*}=1000 G$.

Three different states are clearly indentified; I- for fields lower than than $1000 G$, where there is no reorientation of the surface layer. The reorientation takes place only in the bulk and it is a reversible process. The state IIГcorresponds to a critical state $\Gamma$ where the final orientation is uniform $\Gamma$ with the orientation of the director in some intermediate direction between the initial one and the one imposed by $\mathbf{H}$. The reorientation is a non reversible process. Finally the state IIITcorresponds to a uniform orientation with the director parallel to $\mathbf{H}$ Гeverywhere in the sample. The reorientation induced by the magnetic field is also a non reversible process.

According to the dry-friction model $\Gamma$ only one critical state was expected $\Gamma$ the state II. However we observe that there are two critical states. The discontinuity between the states II and IIIIseems to indicate that there is no more an elastic coupling between the surface layer and the bulk.

We will reanalyse this problemГconsidering that $\Gamma$ in addition to the dry-friction like interaction at the surface $\Gamma$ there is also an elastic interaction $\Gamma$ given by the Rapini-Papoular expression. Therefore $\Gamma$ the torque in the surface due to the surface interactions can be written as:

$$
\Gamma_{S}=\frac{d F_{S}}{d \Phi}=\frac{w}{2} \sin (2 \Phi)+\Gamma_{d}
$$

where $w$ is the anchoring strength of the anchoring energy. The equilibrium at the surface is obtained by the balance of the surface and elastic torques:

$$
\Gamma_{d}+\frac{w}{2} \sin (2 \Phi)=\frac{K}{\xi} \sin \left(\theta-\varphi_{0}\right) .
$$

When the applied torque $\Gamma$ is equal to the critical torque $\Gamma_{C}$ Tthe director in the surface is still parallel to $\mathbf{n}_{0}$, and $\Phi=0$. It results that the critical torque is still given by eq. 14 . However the dependence of $\Phi$ on $H$ Гis:

$$
\frac{1}{\xi}=\frac{(w / 2 K) \sin 2 \Phi+\left(1 / \xi^{*}\right) \sin \theta}{\sin (\theta-\Phi)} .
$$

This equation shows that for $\mathrm{H} \rightarrow \infty(\xi \rightarrow 0)$, leads to $\Phi \rightarrow \theta$. If $H$ is removed $\Gamma$ the final state is stable and homogeneous $\Gamma$ along $\Phi$ (given by the above equation) if the elastic torque in the surface is smaller than the critical one;

$$
\frac{w}{2} \sin 2 \Phi<\Gamma_{C} .
$$

If the above condition is not fulfilled $\Gamma$ the final state is not homogeneous and the final orientation will be along $\Phi^{\prime}$ given by:

$$
\frac{w}{2} \sin 2 \Phi^{\prime}=\Gamma_{C} .
$$

According to this model $\Gamma$ if $H>H_{C}^{*}$, the final state is a critical state $\Gamma$ and the orientation of the director in the surface does not return to the initial orientation if the field is removed. For $H<H_{C}^{*}$, there is no reorientation of the director in the surface layerГonly in the bulk. In this case if the field is removed $\Gamma$ there is a relaxation in the bulk $\Gamma$ and the initial orientation is recovered; the system behaves as if there was a strong anchoring.

From the experimental curve $\Gamma$ one can obtain the surface critical field $\Gamma \mathrm{H}_{C}^{*}=(1000 \pm 100) G$, and from 
the threshold condition. eq.145one calculates the critical torque; $\Gamma_{C}=7 \times 10^{-5} \mathrm{erg} / \mathrm{cm}^{2}$. The experimental curve of $\Phi(H)$ can be fitted $\Gamma$ using eq. $19 \Gamma$ taking $w$ as the fitting parameter. The fitted curve is presented in Fig. 6b $\Gamma$ for a value of $w=8 \times 10^{-5} \mathrm{erg} / \mathrm{cm}^{2} \Gamma$ which corresponds to a weak anchoring strength. However $\Gamma$ the saturation regime $\Gamma \Phi=\theta \Gamma$ occurs with a jump in $\Phi(H) \Gamma$ and not continously $\Gamma$ as could be expected from the model developed above. Moreover $\Gamma$ the saturation would occur for very high magnetic fields.

\section{Conclusions}

In conclusion $\Gamma$ the specific properties of the lyotropic liquid crystals with biaxial deformable micelles can lead to the formation of the biaxial surface layer. The model discussed above shows that the dynamic behavior can be explained $\Gamma$ if we assume a direct interaction of the surface layer with the magnetic field. It is also shown that the magnetic field induces the reorientation of the surface layer leading to a critical state. This reorientation process can be non reversible for magnetic fields higher than the surface critical field $H_{C}^{*}$. The value of $H_{C}^{*}$ can be experimentally determined. To explain the existence of the surface critical field we introduced in the surface energy a "dry friction-like" energy related to the complex molecular interactions between the liquid crystal and the substrates. Despite the aproximations introduced in the calculations $\Gamma$ there is a good accordance with the experimental observations. The second critical state $\Gamma$ with $\mathbf{n}$ parallel to $\mathbf{H} \Gamma$ seems to be related to the breaking of the elastic coupling between the surface layer and the bulk. This problem is being investigated; the third state would exist when the torque at the interface between the bulk and the surface layer overcomes a critical value [24]..

Finally $\Gamma$ for further detailed description of the reorientational behavior of the biaxial surface layer one needs to determine the phenomenological constants introduced in the expansion of the free energy (eq. 1). The structure of the tail of the transmittance curve $(t>>\tau)$ can bring a part of this information[25].

\section{References}

[1] P. G. de Gennes and J. Prost, The physics of liquid crystals, Clarendon Press, Oxford (1991).

[2] C. Rosenblatt and N. M. Amer, Appl. Phys. Lett. 36, 432 (1980).

[3] J. P. Nicholson, J. Phys. (Paris) 48, 131 (1987).

[4] J. Seltzer, P. Galatola and G. Barbero, Phys. Rev. E 55, 477 (1997).

[5] J. Fünfshilling, Cond. Matt. News 1, 12 (1991).

[6] B. Jerôme, Rep. Prog. Phys. 54, 391 (1991).

[7] A. Rapini and M. Papoular, J. Phys. (Paris) Colloq. 30, C4-54 (1964).

[8] K. H. Yang and C. Rosenblatt, Appl. Phys. Lett. 43, 62 (1983).

[9] T. J. Sluckin and A. Poniewierski, Phys. Rev. Lett. 55, 2907 (1983).

[10] H. Yokohama and H. van Sprang, J. Appl. Phys. 57, 4520 (1985).

[11] J. Stelzer, L. Longa and H-R. Trebin, Phys. Rev. E 55, 7085 (1997).

[12] J. D. Parson, Phys. Rev. Lett. 41, 877 (1978).

[13] S. Naemura, Mol. Cryst. Liq. Cryst. 68, 183 (1981).

[14] M. A. Osipov, T. J. Sluckin and S. J. Cox, Phys. Rev. E 55, 464 (1997)

[15] G. Barbero and G. Durand, J. Phys.(Paris), Colloq 47, 2129 (1986).

[16] E. A. Oliveira, A. M. Figueiredo Neto and G. Durand, Phys. Rev. A 44, R825 (1991).

[17] R. F. Turchiello and E. A. Oliveira, Phys. Rev. E 54, 1618 (1996).

[18] M. C. Holmes and J. Charvolin, J. Phys. Chem. 88, 810 (1984).

[19] A. M. Ribas, L. R. Evangelista, A. J. Palangana, E. A. Oliveira, Phys. Rev. E 51, R5204 (1995).

[20] Y. Galerne and J. P. Marcerou, Phys. Rev. Lett.51, 2109 (1983).

[21] V. L. Lorman, E. A. Oliveira and B. Metout, Phys. Lett. A 231, 283 (1997).

[22] E. A. Oliveira, G. Barbero, A. M. Figueiredo Neto and A. K. Zvezdin, Phys. Lett. A 222, 113 (1996).

[23] L. M. Vega, J. J. Bonvent, G. Barbero and E. A. Oliveira, Phys. Rev. E 57, R3715 (1998).

[24] A. L. Alexe-Ionescu, L. M. Vega, J. J. Bonvent and E. A. Oliveira, to be published.

[25] V. L. Lorman, E. A. Oliveira and B. Metout, to be published. 